Notre Dame Journal of Formal Logic

Volume 23, Number 4, October 1982

\title{
A Relational Representation of Quasi-Boolean Algebras
}

\author{
J. MICHAEL DUNN*
}

1 Introduction We follow Białynicki-Birula and Rasiowa [2] in defining a quasi-Boolean algebra to be a structure $(A, \vee, \vee, \wedge, \sim)$, where $(A, \vee, \wedge)$ is a distributive lattice with greatest element $V$ and $\sim$ is a unary operation on $A$ which is an involution, i.e.,

(1) $\sim \sim a=a$

(2) $\sim(a \vee b)=\sim a \wedge \sim b$.

It is easy to verify that setting $\Lambda=\sim V$ gives a least element, and that (2) may be replaced with either of

$\left(2^{\prime}\right) \quad \sim(a \wedge b)=\sim a \vee \sim b$, or

( $\left.2^{\prime \prime}\right) \quad a \leqslant b$ only if $\sim b \leqslant \sim a$.

Essentially identical structures have been investigated by Moisil and Monteiro (cf. [6]) under the name "de Morgan lattices" and by Kalman under the name "distributive $i$-lattices," except Moisil, etc., did not require the existence of a greatest (and least) element. But trivially a de Morgan lattice can always just have these elements appended (if it doesn't already have them), and so we do not bother to distinguish work done on these technically two different kinds of structures. De Morgan lattices have been discussed widely in the literature on constructive logic with strong negation (cf. [6]), and in the literature on relevance logic (cf. [1]), where their representation theory can be thought of as playing a role in the semantics of (first-degree) relevant implication (cf. [4] for a discussion of the relationship of the competing Routley and Dunn semantical treatments of negation to such representation theorems).

*I wish to thank the referee for his suggestions and corrections. 
Various interesting representation theorems have been given for de Morgan lattices, or quasi-Boolean algebras to return to our preferred terminology here. A summary, showing that they are all effectively equivalent can be found in [3] (cf. also [6]). An early and central one of these is due to BiałynickiBirula and Rasiowa and goes as follows. Let $U$ be a nonempty set and let $g: U \rightarrow U$ be such that it is of period two, i.e.,

(3) $g(g(x))=x$, for all $x \in U$.

(We shall call the pair $(U, g)$ an involuted set-g is the involution, and is clearly 1-1.) Let $Q(U)$ be a lattice of subsets of $U$ (closed under $\cap$ and $U$ ) closed as well under the operation of "quasi-complement"

(4) $\sim X=U-g[X] \quad(X \subseteq U)$.

$(Q(U), U, \cup, \cap, \sim)$ is called a quasi-field of sets and is a quasi-Boolean algebra.

Representation theorem of Białynicki-Birula and Rasiowa Every quasiBoolean algebra is isomorphic to a quasi-field of sets.

Although this representation is highly informative, it must be counted as somewhat artificial. It is the purpose of this paper to give a new, more "natural" representation.

2 Representation as relations One way to try to find a more natural representation is to think of naturally occurring transformations $g$ of period two. One that comes quickly to mind is the component-interchange transformation on ordered pairs:

$$
(\overline{x, y})=(y, x) \text {. }
$$

This gives rise to the induced transformation of ordinary conversion on relations (sets of ordered pairs):

(6) $\widetilde{R}=\{(\overline{x, y}):(x, y) \in R\}=\{(y, x):(x, y) \in R\}$.

Indeed, let $U$ be a set, $\rho$ a fixed symmetric relation on $U\left(\rho \subseteq U^{2}\right)$, and let $Q(U, \rho)$ be a lattice of subsets of $\rho$ closed under the operation

(7) $\sim R=\rho-\overparen{R} \quad(R \subseteq U \times U)$.

Let us call $(Q(U, \rho), \rho, \cap, \cup, \sim)$ a quasi-Boolean algebra of relations. Clearly this terminology is justified, since obviously every quasi-Boolean algebra of relations is a quasi-field of sets, and hence a quasi-Boolean algebra. We show that the converse is also true (up to isomorphism).

Theorem 1 Every quasi-Boolean algebra $(A, V, \cup, \cap, \sim)$ is isomorphic to a quasi-Boolean algebra of relations.

Proof: Our proof will be based upon the Representation of Białynicki-Birula and Rasiowa stated above. Let

(8) $f: A \rightarrow P(U)$

be the isomorphism of Białynicki-Birula and Rasiowa, with $g$ the given map of 
period two. Our desired isomorphism sends each $a \in A$ to $g$ restricted to $f(a)$, in symbols:

$$
h(a)=g \uparrow f(a)=g \cap(f(a) \times \operatorname{Rng}(g))=g \cap(f(a) \times U) .
$$

It will turn out that the $h$-image of $A$ is a quasi-Boolean algebra of relations $Q(U, g)$.

We first verify that $h$ is a homomorphism. Thus: ${ }^{1}$

$$
\begin{aligned}
g \uparrow f(a \vee b) & =g \uparrow(f(a) \cup f(b)) \\
& =(g \uparrow f(a)) \cup(g \uparrow f(b))
\end{aligned}
$$

and so $h$ preserves $\vee$. That $h$ preserves $\wedge$ is similar.

As for $h$ preserving ,

$$
h(\sim a)=g \uparrow f(\sim a)
$$

$$
\begin{aligned}
& =g \uparrow(U-g[f(a)]) \\
& =(g \uparrow U)-g \uparrow(g[f(a)]) \\
& =g-g \uparrow(g[f(a)])
\end{aligned}
$$

[ $f$ is an isomorphism]

[Thm. 32 of [7]]

$[\operatorname{Dom}(g)=U]$

Let us leave this dangling to verify the following

Lemma Let $g$ be any 1-1 function. Then

$g \uparrow A=g \uparrow g[A]$

Verification: $(x, y) \in \overline{g \uparrow A} \Leftrightarrow(y, x) \in g \uparrow A \Leftrightarrow y \in A$ and $g(y)=x \Leftrightarrow$ (since $g$ is 1-1) $x \in g[A]$ and $g(y)=x \Leftrightarrow x \in g[A]$ and $(x, y) \in g \Leftrightarrow(x, y) \in g \nmid g[A]$.

Returning now to the main thrust,

$$
\begin{aligned}
\sim h(a) & =g-g \uparrow f(a) \\
& =g-g \uparrow g[f(a)] \\
& =g-g \uparrow g[f(a)]
\end{aligned}
$$

Finally, $h(\sim a)=\sim h(a)$ results from (15) and (19).

As for $h$ being one-one, if $a \neq b$, then $f(a) \neq f(b)$ since $f$ is known to be one-one. Supposing without loss of generality that $x \in f(a)$ while $x \notin f(b)$, then $(x, g(x)) \in g \uparrow f(a)$, but $\notin g \uparrow f(b)$. So $h(a) \neq h(b)$, as desired.

Remark: Clearly the representation would be even "more natural" if in the definition of a quasi-Boolean algebra of relations we could always require that $\rho=$ the universal relation $U^{2}$. This cannot be done, as the reader can easily verify considering the three-element quasi-Boolean algebra defined on $\{-1,0,+1\}$ with the usual ordering and with $\sim a=-a$. One problem is that since $\sim 0=0$, there must be some relation $Z$ (the image of 0 ) so that

(20) $U^{2}-\breve{Z}=Z$, i.e.,

(21) $(b, a) \notin Z \Leftrightarrow(a, b) \in Z$ (for all $a, b \in U$ ).

But since $U$ must be nonempty (having at least three relations on it), there exists $a \in U$, so

$$
(a, a) \notin Z \Leftrightarrow(a, a) \in Z,
$$

an impossibility. 
3 Connections to relation algebras There is another way of algebraizing quasi-Boolean algebras which is already implicit in the Representation Theorem of Białynicki-Birula and Rasiowa, and which is reasonably explicit in Meyer's [5], but seems to receive new meaning set in the context of our Theorem 1 . The idea is that

$$
\sim a=-(a *)
$$

where $*$ is an automorphism of period two on some underlying Boolean algebra and - is the Boolean complement. What reflection on Theorem 1 contributes is the idea that we should regard * as an abstract converse operation.

Being more explicit we define a converse algebra to be a structure $(B, V$, $\wedge, \vee,-, *)$, where $(B, V, \wedge, \vee,-)$ is a Boolean algebra and * satisfies

$$
\begin{aligned}
& (a \wedge b)^{*}=a^{*} \wedge b^{*},(a \vee b)^{*}=a^{*} \vee b^{*},(-a)^{*}=-\left(a^{*}\right) \\
& a^{* *}=a .
\end{aligned}
$$

It is an easy verification that with $\sim$ defined on a converse algebra by (23) we obtain a quasi-Boolean algebra $(B, V, \wedge, \vee, \sim)$. And of course since quasi-Boolean algebras are equationally definable, all of its subalgebras are quasi-Boolean algebras as well. Theorem 1 (or less vividly, the Representation Theorem of Białynicki-Birula and Rasiowa) says that (up to isomorphism) all quasi-Boolean algebras may be obtained as such subalgebras.

All of this suggests the project of providing a natural representation for converse algebras. ${ }^{2}$ To this end we define a converse algebra of relations (a concrete converse algebra) to be a structure $(C(U, \rho), \rho, \cap, \cup,-, \smile)$, where $C(U, \rho)$ is a field of subsets of $\rho \subseteq U^{2}$ (- is complement relative to $\rho$ ) and $\smile$ is the ordinary converse operation on the relations in $C(U, \rho)$. We can now state

Theorem 2 Every converse algebra is isomorphic to a converse algebra of relations.

Proof: A more or less standard Stone-style construction, and all details are left to the reader. $U=$ the set of maximal filters of $B, \rho=\left\{\left(M,[M]^{*}\right): M \in U\right\}$, and for $a \in B$, we define the isomorphism

$$
h(a)=\left\{\left(M,[M]^{*}\right): a \in M\right\}
$$

\section{NOTES}

1. Theorems 30-32 of [7] Ch. 3, saying $f \uparrow(A \cup B)=(f \uparrow A) \cup(f \uparrow B)$, and similarly for $A \cap B$ and $A-B$, are key in the following verifications.

2. It is perhaps worth noting that converse algebras are just the usual algebras of relations minus the operation of relative product, and that there are well-known difficulties (Tarski, Lyndon) with representing the full-relation algebras.

\section{REFERENCES}

[1] Anderson, A. R. and N. D. Belnap, Jr., Entailment: The Logic of Relevance and Necessity, Vol. 1, Princeton University Press, Princeton, New Jersey, 1975. 
[2] Białynicki-Birula, A. and H. Rasiowa, "On the representation of quasi-Boolean algebras," Bulletin of the Polish Academy of Sciences, Cl. III, 5 (1957), pp. 259-261.

[3] Dunn, J. M., "The effective equivalence of certain propositions about de Morgan lattices," The Journal of Symbolic Logic, vol. 32 (1967), pp. 433-434.

[4] Dunn, J. M., "Intuitive semantics for first-degree entailments and 'coupled trees'," Philosophical Studies, vol. 29 (1976), pp. 149-168.

[5] Meyer, R. K., “A Boolean valued semantics for R,” Canberra, typescript (1976).

[6] Rasiowa, H., An Algebraic Approach to Non-Classical Logics, North-Holland Co., Amsterdam, 1974.

[7] Suppes, P., Axiomatic Set Theory, D. Van Nostrand Co., Inc., Princeton, New Jersey, 1960.

Department of Philosophy

Indiana University

Bloomington, Indiana 47405 